\section{Statistical Properties of Height of Japanese Schoolchildren}

\author{
Hiroto KUNINAKA *, Yu Mitsuhashi and Mitsugu \\ Matsushita
}

Department of Physics, Chuo University, Kasuga, Bunkyo-ku, Tokyo 112-8551

(Received November 14, 2018)

KEYWORDS: height, lognormal distribution, normal distribution, multiplicative process, data analysis

The height and weight are the fundamental indices of human growth, which are strongly affected by various factors such as genetic effects, race, nutrition, congenital disease, and so on. The relationship between the indices and physical conditions has been extensively studied to establish a simple measure, such as the body mass index (BMI). ${ }^{1-3)}$ One of the serious health problems is obesity which causes various diseases such as cardiovascular disease and diabetes. Thus, investigating the statistical properties of height and weight from the viewpoint of the human growth process is important for the prevention of adult diseases.

For the statistical property of height, it has been commonly believed that the height distribution obeys the normal distribution, ${ }^{4,5)}$

$$
n(x)=\frac{1}{\sqrt{2 \pi} \sigma} \exp \left[-\frac{(x-\mu)^{2}}{2 \sigma^{2}}\right],
$$

where $n(x)$ is the frequency divided by the total number of individuals, $x$ is the height, and $\mu$ and $\sigma$ are the average and the standard deviation, respectively. If the height distribution were approximated by the normal distribution, it would suggest that the growth of individuals is governed by the stochastic additive process because the sum of independent random variables approximately obeys the normal distribution according to the central limit theorem.

On the other hand, the multiplicative process is often introduced to explain the growth process of living organisms. In the multiplicative process, the growth of living organisms is governed by the time evolution,

$$
\begin{aligned}
X_{t+1} & =\alpha_{t} X_{t} \\
& =\alpha_{t} \cdot \alpha_{t-1} \cdots \alpha_{0} \cdot X_{0},
\end{aligned}
$$

where $X_{t}$ and $\alpha_{t}$ are the size and the growth rate at time $t$, respectively. If $\alpha_{t}$ is independent of the size $X_{t}$, this process is called Gibrat's process, which reproduces the lognormal distribution of $X_{t}$ as

$$
f\left(X_{t}\right)=\frac{1}{\sqrt{2 \pi \sigma^{2}} X_{t}} \exp \left[-\frac{\left[\ln \left(X_{t} / T\right)\right]^{2}}{2 \sigma^{2}}\right]
$$

after long-time growth. ${ }^{6)}$ Here, $f(x), \sigma$, and $T$ are the frequency divided by the total number of individuals, the standard deviation, and the median, respectively. In natural and social phenomena, the lognormal distribution can be observed in various cases such as population distribution of villages in Japan, ${ }^{7}$ ) fragmentation of glass rods, ${ }^{8)}$ and so on.

In this short note, we investigate the height distribution of Japanese schoolchildren by data analysis. Our data analysis is based on the data of the school health survey by the ministry of education, culture, sports, science and technology, Japan (MEXT). ${ }^{9)}$ This survey is performed yearly for the schoolchildren ranging from 5 to 17 in age who belong to the schools randomly sampled from all the schools in Japan. The survey date ranges from April 1 to June 30 in each year. In 2006, the numbers of sampled schools and children were 7755 and 695600, respectively. The data obtained from MEXT are histogram data classified into some classes of height. In the case of 17-year-old female students in 2006, the range of the data, from $130 \mathrm{~cm}$ to $184 \mathrm{~cm}$, are splitted into 55 equal-sized $(1 \mathrm{~cm})$ classes. Note that the interpretation of the data are subject to sampling errors because of the sample survey.

The procedure of our analysis is as follows. First, for the data of each survey year, we fit the height distribution in each age by the lognormal and the normal distributions, respectively, by the use of GNUFIT routine implemented within GNUPLOT, which is based on the Marquardt-Levenberg algorithm for fitting. ${ }^{10)}$ Second, to determine which distribution can be better approximation, we calculate the sum of squared residuals from the data classified into $m$ classes as

$$
R_{i}^{2}=\sum_{j=1}^{m}\left(O_{i}^{j}-E_{i}^{j}\right)^{2},
$$

where $O_{i}^{j}$ and $E_{i}^{j}$ are the observed and the estimated values for the $j$-th height class at each age $i$, respectively. Third, from the sums of squared residuals calculated from both the lognormal and the normal distributions, we calculate the ratio

$$
\epsilon_{i}=\frac{R_{i}^{(L N) 2}}{R_{i}^{(N) 2}},
$$

where $R_{i}^{(L N) 2}$ and $R_{i}^{(N) 2}$ are the sum of residuals calculated from the lognormal and the normal distributions, respectively. The height distribution is well approximated by the normal distribution when $\log \epsilon_{i}$ is larger than 0 , while it is well approximated by the lognormal distribution when $\log \epsilon_{i}$ is less than 0 . We calculate $\epsilon_{i}$ for each age $i$ to obtain the relationship between $\epsilon_{i}$ and age $i$ in a given survey year.

Figure 1(a) shows the relationship between age $i$ and $\log \epsilon_{i}$, where $\log$ is common logarithm, $\log \equiv \log _{10}$. Here we plot the results obtained from the data of both male and female students in 2006. We find the clear transition from the lognormal distribution to the normal distribution in both male and female students around 11 or 13 years old. The timing of the transition is earlier in female students rather than that in male students. We commonly call this period puberty in which physical changes occur in a child's body to become an adult body capable of reproduction. For the adult, the height distribution seems to be approximated by both the lognormal 

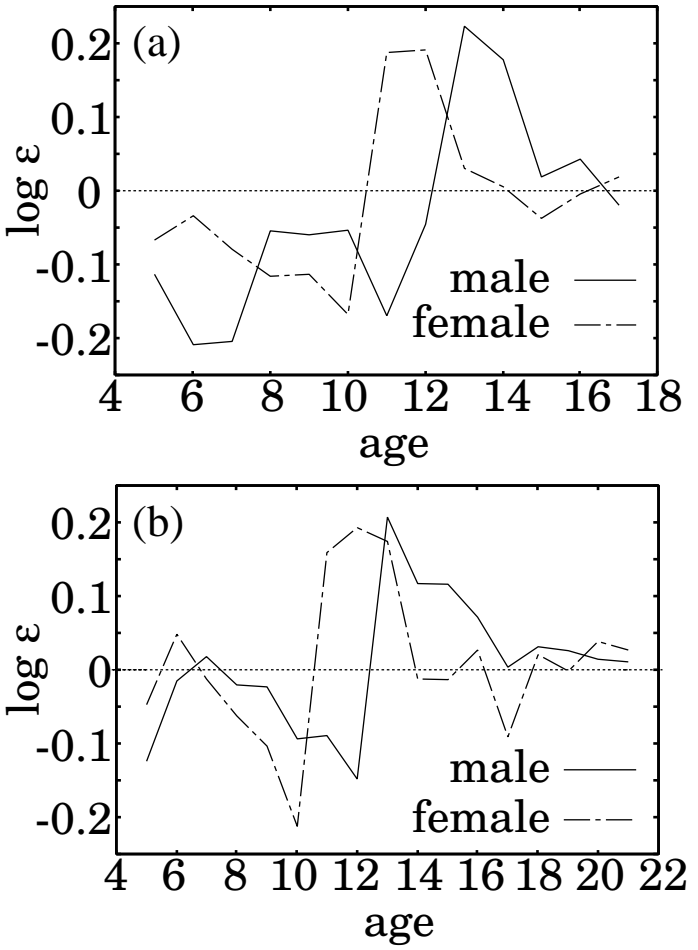

Fig. 1. Relationship between age and $\log \epsilon$ in (a) 2006 and (b) 1970. Solid and chain lines are for male and female students, respectively.

and the normal distributions. Limpert et al. pointed out that the similar tendency can be seen in the height distribution of female adults. ${ }^{11)}$

In the case of another year, Fig. 1(b) shows the result in 1970, where we also find clear transition from the lognormal distribution to the normal distribution although the timing of the transition is slightly different from that in 2006. These results would imply that the transition from the lognormal distribution to the normal distribution in height distribution of schoolchildren occurs in any period in Japan.

Next, we investigate the time evolution of the height distribution of the cohort born in 1990. We analyze the data ranging from 1995 to 2007 . Notice that all the members belonging to the cohort are totally different because the sampled schools and individuals are different according to the survey year. Figure 2 shows the relationship between $\log \epsilon$ and age of the male and female schoolchildren who belong to the cohort. Also in this case, we can observe the similar transition from the lognormal distribution to the normal distribution. After the transition occurs, around 16 years old, the data can fit both distributions.

From these analyses, we conjecture that the growth process of individuals obeys the multiplicative process before puberty. When an individual reaches the age around puberty, the growth process becomes rather additive, which might cause the transition of the height distribution to the normal distribution. Modeling the growth process to reproduce such a transition is our near future task. Besides the modeling, we need to analyze the data of other countries to investigate whether similar tendency

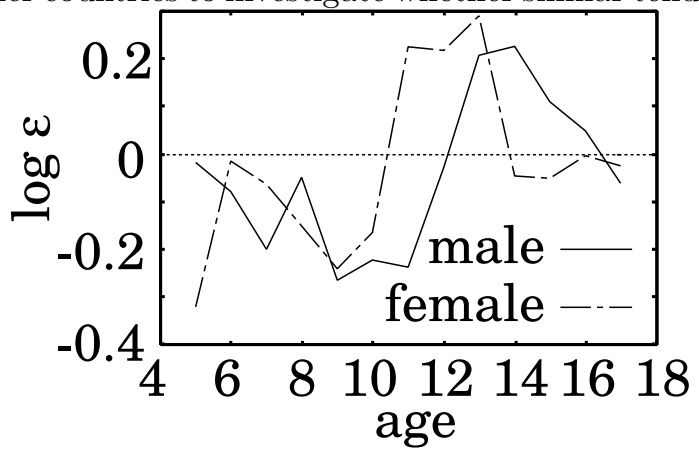

Fig. 2. Relationship between age and $\log \epsilon$ for the cohort born in 1990. Solid and chain lines are for male and female students, respectively.

can be universally observed among schoolchildren. In addition, the growth process of height is closely related to that of weight. We will report our analysis of the weight distribution in another occasion.

\section{Acknowledgement}

HK would like to thank K. Kuninaka and M. Niidome for their useful advices. This work is supported by a Grant-in-aid from MEXT, Japan (Grant No. 18340115).

1) G. Eknoyan: Nephrol. Dial. Transplant, 23 (2008) 47.

2) T. Khosla and C. R. Lowe: Brit. J. Prev. Soc. Med., 21 (1967) 122.

3) A. Quetelet: A Treatise on Man and the Development of his Faculties (Burt Franklin, New York, 1968) Reprinted., originally published in 1842

4) T. Clemons and M. Pagano: Am. Stat. 53 (1999) 298

5) B. A'Hearn, F. Peracchi, and G. Vecchi: Res. Paper. Ser. CEIS Tor Vergata, 6 (2008).

6) E. W. Crow and K. Shimizu: Lognormal Distribution: Theory and application (Marcel Dekker, New York, 1988).

7) Y. Sasaki, H. Kuninaka, N. Kobayashi, and M. Matsushita: J. Phys. Soc. Jpn. 76 (2007) 074801.

8) T. Ishii and M. Matsushita: J. Phys. Soc. Jpn. 61 (1992) 3474

9) http://www.mext.go.jp/.

10) W. H. Press, et al: Numerical Recipes in Fortran 2nd. edition (Cambridge University Press, Cambridge, 1999).

11) E. Limpert, W. A. Stahel, and M. Abbt: BioSci., 51 (2001) 341. 